\title{
Challenges of the patient transition process from the intensive care unit: a qualitative study
}

\author{
Kobra Ghorbanzadeh ${ }^{1,2}$, Abbas Ebadi ${ }^{3}$, Mohammadali Hosseini ${ }^{4}$, Sadat Seyed Bagher Madah ${ }^{4}$, \\ Hamidreza Khankeh ${ }^{5,6}$
}

${ }^{1}$ Ph.D. Candidate in Nursing, Department of Nursing, University of Social Welfare and Rehabilitation Sciences, Tehran; ${ }^{2}$ Department of Nursing, Khalkhal University of Medical Sciences, Khalkhal; ${ }^{3}$ Behavioral Sciences Research Center, Lifestyle Institute, Nursing Faculty, Baqiyatallah University of Medical Sciences, Tehran; ${ }^{4}$ Department of Nursing, University of Social Welfare and Rehabilitation Sciences, Tehran; ${ }^{5}$ Health in Emergency and Disaster Research Center, University of Social Welfare and Rehabilitation Sciences, Tehran, Iran; ${ }^{6}$ Department of Clinical Science and Education, Karolinska Institute, Stockholm, Sweden

Background: The transition of patients from the intensive care unit (ICU) to the general ward is challenging. This study aimed to explain the challenges that patients face during the transition process.

Methods: In this qualitative research of conventional content analysis, data collection was conducted between February 2018 and July 2019 in educational hospitals. After obtaining informed consent, purposive sampling was performed with 22 nurses, intensive care physicians, anesthesiologists, and patients and their families using in-depth semi-structured interviews until data saturation.

Results: The content analysis yielded three main themes in the challenges patients face during the transition process from the ICU: mixed feelings regarding transition (happiness/hope, worry/uncertainty, abandonment); care break (different atmosphere, the difference between the program and the quality of care, assigning care to the patient and family, and care culture and beliefs); and search for support and information (ineffective communication, selfcare capacity of patient and family, ineffective and disrupted training, and weak follow-up programs), which inflicts care shock in the patients.

Conclusions: The results showed that patients and their families were in a state of care shock during the ICU transition process and were sometimes disconcerted. It is necessary to design and implement care models according to the needs and challenges patients face during the transition period from ICU (patient-centered), based on the evidence available, and after considering the field of medicine and the accessibility of care in the country. The transition process can be improved and enhanced by obtaining knowledge about ICU care and related challenges as well as organizing a learning environment.

Key Words: intensive care unit; patient transfer; transition care; qualitative research

\section{INTRODUCTION}

The patient transition from the intensive care unit (ICU) to the general wards of a hospital is a complex process with many potential challenges [1]. It is also one of the most vulnerable transitions patients face [2]. Patients admitted to the ICU may experience several complex

\section{Original Article}

Received: August 8, 2020

Revised: November 11, 2020

Accepted: November 23, 2020

Corresponding author Mohammad Ali Hosseini Department of Nursing, University of Social Welfare and Rehabilitation Sciences, Tehran 098, Iran Tel: $+90-9121488457$ Fax: +90-22180036

E-mail:mahmaimy2020@gmail.com

Copyright @ 2021 The Korean Society of Critical Care Medicine

This is an Open Access article distributed under the terms of Creative Attributions Non-Commercial License (https:/I creativecommons.org/li-censes/by-nc/4.0/ which permits unrestricted noncommercial use, distribution, and reproduction in any medium, provided the original work is properly cited. 
transition issues simultaneously. These transitions can be related to their health-disease status, their situation, and their organization. Specifically, these include the transition from health to a life-threatening illness and vice versa; the transition from specialized, technical, and individual care to general care; the transition from a secure environment inside the ICU to a vulnerable and unpredictable environment; and finally the transition from despair to independence $[3,4]$.

The word transition is used to describe a process of change in the developmental stages of life, or alterations in health and social circumstances rather than responses to change [5]. The transition is associated with changes in identity, role, communication, ability, and behavior, for adaptation to which an individual requires new knowledge, behavior change, and selfdefinition [6]. Factors that can affect the transition process include preparation and planning, individual knowledge, meaning and experience of the transition, individual's environment in transition, and, finally, the emotions and physical well-being of the people experiencing the transition [7].

ICU patients recovering from a critical illness often suffer from physical and mental complications that require recovery support. Therefore, proper patient preparation throughout the health-disease transition is critical for patients and their families [8]. Although ICU discharge is a positive step and physical improvement for the patient, a patient who is not psychologically ready to be transferred to the ward will experience severe anxiety during the transition from the ICU to the ward [9]. The transition of the patient from the ICU is a period of vulnerability in terms of medical errors which can lead to unwanted complications, increased costs, and patient dissatisfaction. This may be related to various factors such as the high number of patients with complex needs, reduced monitoring outside the ICU, staff with different competencies and skills, and communication failure [10]. How the transition from the ICU to the hospital ward is experienced by the patient and the family is influenced by how it is organized, communicated, and executed [11].

Anxiety and unpreparedness can lead to a lack of understanding and readiness for the self-care role in other areas [12]. Transition stress is a mental disorder that the patients experience when they move from one environment to another during which they need to be psychologically prepared to gradually break away from equipment and constant monitoring [13]. This stress will have long- and short-term manifestations. A nurse must reduce the transition stress for both the patients and their families using certain strategies such as managing the time of transition, requesting that patients and their fami-

\section{KEY MESSAGES}

- The challenges patients and their families experience in the transition process were considered.

- It is necessary to design and implement care models according to the needs and challenges patients face during the transition period (patient-centered), based on the evidence available.

- The transition process can be improved and enhanced by obtaining knowledge about intensive care unit transition care and related challenges as well as organizing a learning environment.

lies ask questions, and training and educating them in relation to clinical progress and any forthcoming issues [14].

Healthcare providers recognize that successful transitions in care require effective multi-disciplinary communication, comprehensive planning, and shared accountability throughout the entire transition process [15]. Poor management of the transition process has negative effects on the patient's transition, health, treatment goals, and outcomes, and increases problems such as prolonged hospital stay, high cost of care, greater degree of disability, and psychological stress for the patient and family, and ultimately unavoidable death. In contrast, there is new evidence that organized care in the transition process has shown beneficial results for patients and their families that include greater satisfaction with care, successful transition, better preparation of the patient for transition and fewer visits to emergency clinics, as well as less need for re-hospitalization of these patients $[1,7]$.

Developing a plan to continue care during the transition period is essential for patients with critical conditions and their families. The start of this program from the ICU is of particular importance [16]. The patient and their family must be involved in the planning process for the transition from the ICU. The long-term follow-up of patients is highly important after passing the critical conditions and transition from the ICU [17]. Therefore, it is essential to understand the experiences of patients and care providers in order to develop protocols to improve patient transition from the ICU to other wards. However, there is a lack of research that explores the challenges patients face when being transferred from the ICU. Thus, it is essential to perform a comprehensive review to identify the challenges facing patients during the transition process from the ICU to other wards and to fill in the existing knowledge gaps with suggestions that can improve this process [18]. This study aimed to investigate the understanding of 
and challenges patients face during their transition process from the ICU.

\section{MATERIALS AND METHODS}

\section{Ethical Considerations}

This study was approved by the Ethics Committee of the University of Social Welfare and Rehabilitation Sciences, Tehran, Iran (IR.USWR.REC.2017.198). Informed written consent was obtained from the participants to participate in the study. The participants were also assured that their conversations would remain confidential and that the audio file would be destroyed after being transcribed.

\section{Research Design}

This qualitative conventional content analysis was part of an extensive study on the patient transition from ICU to other wards. This part of the study was conducted using the content analysis method of Graneheim and Lundman [19].

\section{Setting and Participants}

This study was conducted at adult ICUs and general wards of educational hospitals affiliated with the University of Medical Sciences in Iran. Purposive sampling was used in this study. In this type of sampling, the selection of participants is based on their first-hand experience and knowledge about a phenomenon, and the sampling ends when derived concepts and their relationships are saturated. Data saturation occurs when the researcher does not obtain new data by continued sampling [19].

We interviewed ICU physicians, anesthesiologists $(n=6)$, ICU nurses and ward nurses $(n=8)$, as well as patients and their families $(n=8)$ who were willing to participate and talk about their experiences. Age, sex, and years of working experience were also considered to achieve variation in the data. Health care providers had to have at least 1 year of experience working in their designated environments, given the average age range of 35-45 years for the nurses and physicians, and a clinical experience of 9-12 years. The age of patients was 6275 years, length of stay in ICU was 5-10 days and the main reasons for ICU admission included pneumonia, heart failure, coronary artery bypass graft, and chronic obstructive pulmonary disease. Inclusion criteria for participants was at least 5 days of hospitalization in the ICU with subsequent transfer to a general ward. All interviews were conducted in a prearranged calm and private meeting room in the hospitals.

\section{Data Collection}

Data were collected from February 2018 to July 2019 through 22 semi-structured in-depth interviews conducted by the first author. The interviews were performed in hospitals, digitally recorded, and lasted 30 to 90 minutes. Initially, the researcher talked to each potential participant and, while explaining the purpose and research questions, determined the appropriate time for the interview if he/she was willing to participate.

The interview guide included a few open-ended questions to allow participants to elaborate on their perceptions and experiences and the challenges experienced during the patient transition process from the ICU to the general ward. Semistructured questions were used when necessary to ensure that everyone was given the opportunity to discuss anticipated and newly emerging themes. The main interview questions were, "What complications did you experience during the transition from ICU?" "What difficulties did you experience during the transition from ICU?," "Would you please tell me about your experiences of patient transition from the ICU?," and "May you please tell me about your challenges during the transition of your patient?" Moreover, probing questions were used to further identify the experiences of the participant. Examples of these questions were, "Can you explain more about this?," “Can you give an example of this?," and "What do you mean by ...? Can you clarify what you mean by an example of your personal experiences?" The participants were able to talk freely about their experiences without any restrictions on time. At the end of each interview, participants were asked the question: "Is there anything you have experienced on this subject that I have not queried?" And at the end of each interview, we thanked the participant and said that he/she could call if he/she had any questions about the interview.

The researcher also observed the behavior and interactions of the care providers with patients and colleagues in the ICU for 20 hours. Each observation lasted between 54 and $79 \mathrm{~min}$ utes (median, 64 minutes). Since the researcher was in the ward earlier than the specified time, he did observations before the interview. For the observation, the researcher sat in the nurses' station and observed their activities and interactions, and their verbal and nonverbal behaviors. The researcher took notes during the observation, and after the observation was complete, recorded it anonymously and used it as data. Field notes were also taken during the interview to assist in data analysis and to document concepts for future exploration.

\section{Data Analysis}

To analyze the data from the first interview and in parallel to 
the interviews based on the method by Graneheim and Lundman [19], five steps were proposed for the analysis of the qualitative data as follows. (1) Transcribing the entire interview immediately after each interview. (2) Reading the entire interview text to get a general understanding of its content. (3) Identifying the meaning units and basic codes. (4) Classifying similar codes in the more comprehensive categories. (5) Identifying the latent content in the data. First, all text material such as field notes, memos, and transcribed recorded text was merged, read, and discussed to get a sense of the whole. Firstlevel coding was performed, in which the sentences that answered the questions in the interview were identified. The main concepts in these statements were then coded, and related codes were merged. Next, based on similarities, the classes were eventually extracted and the concepts underlying the data were extracted and named as themes. To validate the collected data, the results of the interview were read repeatedly and the opinions of the colleagues and some participants were frequently used to confirm the data.

\section{Trustworthiness}

The four criteria proposed by Lincoln and Guba [20,21] were used to ensure the trustworthiness of the data. The researcher attempted to increase the credibility and dependability with long-term participation, sufficient involvement, and interaction with participants, gathering valid information, and verifying the information. Repeated step-by-step data collection and analysis as well as the reviews of supervisors, consultants, and experts were used to increase the reliability of the data. To increase conformability and transferability, attempts were made to provide a rich description of the research report for the evaluation and applicability of the research to other fields.

\section{RESULTS}

Maximum variation sampling produced a diverse group of participants. A total of 504 initial codes were extracted from the rich and in-depth descriptions of the participants. After review, the codes were summarized and categorized according to similarity and appropriateness. Three main themes were obtained for the challenges of patient transition from the ICU: (1) Mixed feelings regarding the transition (happiness/ hope, worry/uncertainty, and abandonment), (2) care break (different atmosphere, the difference between the program and the quality of care, assigning care to the patient and family, and care culture and beliefs), and (3) search for support and information (ineffective communication, self-care capac-
Table 1. Challenges of the transition process from the intensive care unit

\begin{tabular}{|c|c|}
\hline Category & Subcategory \\
\hline \multirow{3}{*}{$\begin{array}{l}\text { Mixed feelings regard- } \\
\text { ing the transition }\end{array}$} & Happiness/hope \\
\hline & Worry/uncertainty \\
\hline & Abandonment \\
\hline \multirow[t]{4}{*}{ Care break } & Different atmosphere \\
\hline & $\begin{array}{l}\text { Difference between the program and the } \\
\text { quality of care }\end{array}$ \\
\hline & Assigning care to the patient and family \\
\hline & Care culture and beliefs \\
\hline \multirow{4}{*}{$\begin{array}{l}\text { Search for support and } \\
\text { information }\end{array}$} & Ineffective communication \\
\hline & Self-care capacity of patient and family \\
\hline & Ineffective and disrupted training \\
\hline & Weak follow-up programs \\
\hline
\end{tabular}

ity of patient and family, ineffective and disrupted training, and weak follow-up programs). The classes are described and explained in the following text (Table 1).

\section{Mixed Feelings Regarding the Transition}

Patients who have regained some of their health and have reached the stage of discharge from the ICU experience contradictory feelings. Since the nature of hospitalization in the ICU is critical, the transition from the ICU to the ward is a positive step in recovery for the patients and their families. About happiness/hope for improvement, patient 6 stated: “... I was happy. I wasn't comfortable there was no companion. I was uncomfortable. I wanted water. No one came to help me. I asked the doctor to send me to a ward ... I became very happy. I must obtain the conditions for the transition to a ward. The doctor should diagnose to go to the ward. I told the doctor to send me to the ward, only the ward. Have you ever understood freedom? Because you are in the ICU, it means you have a problem, but when you are transferred you have been screened, i.e., to get rid of all problems. They keep you there to solve the problems".

Fear, worry, and uncertainty were the specific reactions of the patients and their families and the treatment team, who were concerned about the recovery situation, continuing the treatment of the disease, the quality of care, and self-care by the patient and the family. As for feeling neglected and abandoned, the caregiver of patient 8 reported: “... When the patient was transferred, we felt confused, as if we didn't know anything. At 2:30 they brought him to the ward in a wheelchair. After the room was determined, which did not take 
more than 5 minutes, there was nobody to give us advice and training to transfer the patient with good spirit. It is very good that someone teaches us how to do physical exercises. Everyone who comes says do exercise, but we don't know how to do it".

\section{Care Break}

Patients will experience a care break if the transition from the ICU is done without preparation. This may be related to a variety of factors, including the high number of patients with complex needs, reduced monitoring outside the ICU, staff with different competencies and skills, and communication failure. The treatment and care system employees count on the ability of the caregivers in the patient's family. After the transition from the ICU, continuous care is suddenly reduced and left to the patient's family and primary caregivers. There is a considerable physical and psychological pressure that caring places on caregivers. Nursing staff need the support of these caregivers and should encourage them to share their feelings, concerns, and problems so caregivers feel less lonely in the care experience.

In this study, the participants experienced a serious gap in the continuity of care due to the obvious difference in the diversity of modern technologies and nursing staff with a higher level of capability than in the general wards. The experiences of the participants indicated that a huge difference would lead to problems for patients and non-compliance with the new environment. Examples of extracted subthemes in this theme were: the different atmosphere, the difference between the program and the quality of care, assigning care to the patient and family, and care culture and beliefs.

Sometimes there is little access to facilities that meet their basic needs, and unfortunately, in most cases, the shortages were not only related to expensive and advanced hospital equipment but also included the simplest essentials of the patient.

Regarding the difference between the two participatory environments, patient 8 reported: “... The environment changed for me. It was hard for me not to sleep in the morning. There was one nurse for each patient here, but the number of nurses is low. My sister goes and says and then they take care of it ... Our biggest problem is that care has been reduced and we don't have peace of mind. At 1:00 am, the patient's companion comes to the room to use the bathroom, she comes to wash the dishes, she mistakenly thinks it is a hotel. We don't have time to rest. Tonight, I want to write a no entry announcement ..."

Participating nurse 8 talked about the difference between equipment and staff: “... but we don't have enough equipment and monitoring. We only have two monitors in the ward. Many patients do not have a monitor with family caregivers ... In the ward when the patient is ill-transferred the caregivers become nurses to inform us of the patient's conditions and they are complaining about this situation ... We also have problems with oxygen, sometimes it happens that you must take away oxygen from one patient and give it to another patient ... Let me see if they don't have respiratory distress and caregivers tell us if there's a problem ..."

Patient 4 expressed concerns about the persistence of specific care needs: “Now my wound is open and swollen. I don't know what happened. I forgot to tell the doctor. I'm very worried. I'm not in pain, but the cough bothers me ... It is better that a system follows and supports the things I don't know, I have a problem asking her/him. Tell me about my pain. I ask questions, I have a question, I will bring it. Take care ..."

Participating nurse 8 talked about the improper patient-nurse ratio: "... The number of patients is seven or higher. Only when the number is high, the quality is low, we can't take care of it in time, we prioritize ... I have eight patients, but this patient transferred from the ICU needs special care and they want us, but it is not possible, we sometimes miss things. It took me half an hour to receive this patient..."

Some participants in the treatment team considered the prevailing culture to be effective in improving patient experience during the transition period, which sometimes caused problems for the patients. A head nurse talked about the interference of care culture and beliefs: “... Look, a series of things have been institutionalized in the culture of our nation, whatever you do is not right. For example, many are very addicted and it's normal for us to give morphine and methadone. We deliver the substances to the ward we tell them, don't give it to him, he gives it to her secretly, he returns with respiratory arrest conditions. It's people's culture all people claim to be super-specialists. With two internet searches, they think they know a lot. They think they can do anything. I don't know anything to read we have seen this a lot especially aspiration: it's only the interference of caregivers. I acknowledge the shortage of staff. The nurse is also busy. They give him too much oxygen. They get the complete bed rest (CBR) patient, to walk forcibly ..."

About assigning care to the patient and family, patient caregiver 9 said: “If we didn't take care, it wouldn't be good. My son did all the work and dressing, etc. We had a health care house. We spent 500 million rials. My eldest son was getting more..." 


\section{Search for Support and Information}

Patients, their families, and medical staff sought information and support to achieve the desired therapeutic results. In this study, the search for support and information was one of the themes that was extracted from codes: ineffective communication, self-care capacity of the patient and family, ineffective and disrupted training, and weak follow-up programs. The self-care capacity of individuals varies depending on the socioeconomic status, factors influencing learning, and life experiences. The goal of self-care is to minimize the professional care of these patients. Patients experience more physical disabilities and psychosocial problems due to a lack of awareness of the disease, treatment, and self-care.

About the self-care capacity patient 8 stated: “... Now it's just too expensive, the question is if it will be efficient, the patient does not suffer, and disability is accepted; they don't accept it. They say get a loan. We don't have animals, gardens, or land, I manage our life through construction work ... Then, the main caregiver who was the patient's wife said we don't have a car, I came here by car every day and I came back in the evening. Because the ICU didn't allow a companion, I commuted for 6 days. It costs a lot. He himself can't go to work, the kids don't work either. We have rural insurance, we don't get help from anywhere. We first refer to a health center to approve the insurance, they said, we have to see the patient who is hospitalized here..."

Regarding the specific nature of these patients after the transition from the ICU, the caregiver of patient 2 said: “... With such conditions, I am not sure whether my patient has fully recovered so that I can take him home because my patient underwent surgery on 31 April and was hospitalized until August 9, and even now she can't hold a spoon. Now with this situation, I can take my patient home ..."

About ineffective and disrupted training nurse 4 reported: "It's a question of learning ability. Older patients who haven't been able to get an education. It is difficult for them to learn. You want to teach, he says ok, but she forgets an hour later. For example, I tell him to cough, do spirometry, but he forgets about it an hour later. I think it should be taught before the operation. What will be your situation? What is the importance of care? Because she is already prepared, she will accept it more easily later".

Regarding ineffective communication nurse 10 reported: "Because the patient is coming from the ICU, tensions will arise as if a monster is coming. When we go, three or more people get engaged to be mentally drained. If you don't say something, they say you didn't say that. Sometimes we strug- gle and the supervisor gets involved. For example, a damaged vein or a bloody dressing. Some colleagues become emotionally drained. They are not to blame: the workload is high. For instance, you are supposed to take this patient and get a bad patient from the operating room. As if a 100 -member army is waiting to fight".

Patient 1 also commented on the weakness and inadequacy of communication, she stated: "Because communication is disrupted between the patient, the doctor, and the nurse and if they provide a brief explanation, there is no need for entering the ICU. There is no exchange of information, or it is very weak. The main caregiver is an expert in another field and she/he has no medical specialty. It's more comfortable in the ward, we see a doctor while visiting, we ask about our patient's conditions."

\section{DISCUSSION}

This study aimed to get a deeper understanding of the challenges involved in the patient transition process from the ICU to the general ward. Due to recent medical advances, the survival rate of hospitalized patients in the ICU has increased. However, these patients are not without complications. Various studies have shown that these patients suffer from cognitive, psychological, and physical problems [22]. Such disorders lead to medical complications and a decline in patient quality of life after hospitalization in the ICU $[4,23]$.

The results of this study were classified into three main themes according to the challenges of the patient transition process from the ICU, including: (1) mixed feelings regarding the transition (happiness/hope, worry/uncertainty, and abandonment); (2) care break (the different atmosphere, the difference between the program and the quality of care, assigning care to the patient and family, and care culture and beliefs); and (3) search for support and information (ineffective communication, self-care capacity of patient and family, ineffective and disrupted training, and weak follow-up programs). These challenges lead to care shock.

As one of the results of this study, mixed feelings regarding the ICU transition, was a conflicting feeling that existed in patients and their families. The ambiguity between the positive and negative experiences during the transfer from the ICU to the general wards is in line with the studies of Cullinane and Plowright [24] and Cypress [25]. Snow et al. [12] and Op 't Hoog et al. [26] reported that the patients and their families were confused and felt abandoned because of conflicting advice from health care providers and an inability to communicate 
with the health care team for guidance. Forsberg et al. [27] indicated feelings of anxiety, and fear on the part of the patient and the family during the transition to an unfamiliar environment. In the study by Chaboyer et al. [28], the main challenges for patients and their families were feeling insignificant in the context of the ward admitted to and a feeling of duality. Most patients and their families feel insecure during this period [4]. Patients felt that their basic care needs were overlooked and that ongoing support during recovery was inadequate that resulted in feelings of demoralization and depression [29].

In our investigation, patients and their families described the feeling of the care break between the ICU and the general wards. Due to the obvious differences in the diversity of modern technologies and nursing staff with a higher level of competence, there is a serious gap in the continuity of care. The experiences of participants showed that a stark difference led to problems for patients and a mismatch with the new environment. Patients need to be psychologically prepared to gradually separate from equipment and monitoring $[13,30]$. According to Op 't Hoog et al. [26], this difference is less prominent depending on the contrast between the two wards, perhaps, some participants were better at putting the new context into perspective, or they had a different coping style. Patients experienced the shift from being the center of all attention to being just 'one in the crowd'. The ICU patient had to share the attention of fewer staff with other patients with similar needs [11]. In the study by de Grood et al. [18], communication and organizational culture were among the main factors involved in sensations of care break during the patient transition from the ICU to a ward, and the application of multiple communication standards and methods was effective in improving the quality of care.

Moreover, awareness about the existing gap between the level of care provided in the ICU and the general wards should induce the sending and receiving units' healthcare providers to work together and consider an appropriate continuity of care as a shared collective responsibility.

Another result of this study was the search for support and information. Research shows that having the right knowledge and skills can help a person experience transition effectively. The transition period is a difficult moment for the patients. Patients should receive preparations such as training, information, or referral to support resources to facilitate the transition [31]. Lack of proper information can lead to stress, anxiety, and insecurity. Having a supportive environment during the transition creates a positive sense of well-being and improves the transition that one experiences. Patients who do not receive adequate support experience negative emotions, weakness, confusion, frustration, and conflict $[7,32]$.

The experiences of the participants in the study by Forsberg et al. [27] showed that they needed to receive information to be transferred to the general ward. Information is key in the movement toward empowerment. Patients must have information and knowledge of their disease and health to make the best decisions on actions and must be considered throughout the transition from the ICU to the hospital ward [11]. Information and teaching are aimed at relatives with a focus on specific transfer plans, ward information, staff information, expectations in the hospital ward, and support services for the family [33]. Nurses in practice are the instructors of patients during the transition. Nurses develop new skills, knowledge, and behaviors that facilitate the desired outcome of patient transition [34].

Interviews with participants showed that patients and family self-care was effective in a successful transition. The selfcare capacity of each individual varies depending on socioeconomic status, factors affecting education, and life experiences. The goal of self-care is to minimize the professional care of these patients. Patients experience more physical disabilities and psychosocial problems due to a lack of awareness of their disease, treatment, and self-care. The transition can also change the behavior of the person or determine the behavior of the patient in response to the transition, and affect the self-efficacy of the patient [7]. Relatives of the ICU patients highlighted the lack of sufficient physical and neuropsychosocial rehabilitation of the patient after the ICU discharge [35]. The problem of inadequate communication for information exchange among the care providers is the potential source of mortality and morbidity in patients. These communication problems are more common in the transition hand-off between two places when the responsibility of the patient shifts between the members of the care team [36] and if the communication method is not clear and consistent. A basic analysis of these failures showed that the lack of a standard protocol for transition from the ICU to a ward is a contributing factor to the potential risks of harm to the patient [37]. In this study, the findings indicate that ineffective communication is one of the challenges of the patient transition process from the ICU. In previous studies, the challenges associated with this transition included an increased risk of medical errors, unwanted complications, readmission, dissatisfaction with care, and death $[24,38]$.

Our study has several implications for practice that suggest the need to increase safe transitions. First, a care transition 
program should be adopted. Second, improve communication during the transition. Third, specific tools, checklists, and a systematic approach should be used. Fourth, careful planning and adequate support are necessary. Finally, regular critical care transition follow up should be ensured together with the implementation of early plans to promptly detect and treat the deterioration of patients. Health care providers must aim to make the transition less abrupt. Our results also suggest that people experience stressful situations during their transition period.

The patient transition between the ICU and ward is associated with high challenges and risks. The main findings obtained from the experiences of patients and their families in the transition showed that during the transition period, patients underwent care shock in response to the care atmosphere change. It is better to design the care models according to the needs and challenges of patients in this period using the available evidence in the national treatment and care context. We recommend more research on strategies for the continuity of care during patient transitions after an ICU stay. However, the individual and a set of supporting systems are effective determinants in dealing with the transition phenomenon. If the patient transition from the ICU is not performed properly, there are risks to the health of the patient. Health care providers must be aware of the relatives' expectations and proactively ask them for their needs to secure continuity and establish and maintain lines of communication.

A limitation of interpreting the findings of this study is that some problems and challenges may be forgotten due to the critical transition period. Another limitation is that the transition process is affected by various factors. Hence, the views of other caregivers involved in this process could be explored in future studies. Moreover, further studies are needed on new methods of rehabilitation, training, and support to improve the experiences of patients and their families during the transition from the ICU.

\section{CONFLICT OF INTEREST}

No potential conflict of interest relevant to this article was reported.

\section{ACKNOWLEDGMENTS}

This research is part of of Kobra Ghorbanzadeh in nursing at the University of Social Welfare and Rehabilitation Sciences, IR.USWR.REC.2017.198.
The researchers would like to sincerely thank all the officials and staff of the university who helped with this dissertation. We are also grateful to the members of the treatment team who provided the researchers with their excellent cooperation, time, and honesty.

\section{ORCID}

Kobra Ghorbanzadeh https://orcid.org/0000-0003-2113-1432 Abbas Ebadi https://orcid.org/0000-0002-2911-7005 Mohammadali Hosseini https://orcid.org/0000-0001-7457-0602

Sadat Seyed Bagher Madah https://orcid.org/0000-0001-6048-1481

Hamidreza Khankeh https://orcid.org/0000-0002-9532-5646

\section{AUTHOR CONTRIBUTIONS}

Conceptualization: AE. Data curation: KG. Formal analysis: KG, MH. Methodology: KG, MH, HK. Project administration: SSBM. Visualization: MH. Writing-original draft: KG. Writingreview \& editing: KG, MH, HK.

\section{REFERENCES}

1. Rosa RG, Maccari JG, Cremonese RV, Tonietto TF, Cremonese $\mathrm{RV}$, Teixeira C. The impact of critical care transition programs on outcomes after intensive care unit (ICU) discharge: can we get there from here? J Thorac Dis 2016;8:1374-6.

2. Buchner DL, Bagshaw SM, Dodek P, Forster AJ, Fowler RA, Lamontagne F, et al. Prospective cohort study protocol to describe the transfer of patients from intensive care units to hospital wards. BMJ Open 2015;5:e007913.

3. Ramsay P, Huby G, Thompson A, Walsh T. Intensive care survivors' experiences of ward-based care: Meleis' theory of nursing transitions and role development among critical care outreach services. J Clin Nurs 2014;23:605-15.

4. Herbst LA, Desai S, Benscoter D, Jerardi K, Meier KA, Statile $\mathrm{AM}$, et al. Going back to the ward: transitioning care back to the ward team. Transl Pediatr 2018;7:314-25.

5. Kralik D, Visentin K, van Loon A. Transition: a literature review. J Adv Nurs 2006;55:320-9.

6. Wilkins KL, Woodgate RL. Transition: a conceptual analysis in the context of siblings of children with cancer. J Pediatr Nurs 2006;21:256-65.

7. Al-Yateem N, Docherty C. Transition: a concept of significance to nursing and health care professionals. J Nurs Educ 
Pract 2015;5:35-40.

8. Ludin SM, Parker S, Arbon P. A survey of Malaysian Critical Intensive Care Unit nurses' awareness of patients' transition experiences (PE) and transitional care practice (TCP). Intensive Crit Care Nurs 2014;30:196-203.

9. Zakerimoghadam M, Ghyasvandian S, Asayesh H, Jodaki K. Prevalence of anxiety in the process of transferring patients from cardiac surgery intensive care unit to the general ward. Crit Care Nurs J 2016;9:e8001.

10. Stelfox HT, Bastos J, Niven DJ, Bagshaw SM, Turin TC, Gao S. Critical care transition programs and the risk of readmission or death after discharge from ICU. Intensive Care Med 2016; 42:401-10

11. Herling SF, Brix H, Andersen L, Jensen LD, Handesten R, Knudsen $\mathrm{H}$, et al. Patient and spouses experiences with transition from intensive care unit to hospital ward: qualitative study. Scand J Caring Sci 2020;34:206-14.

12. Snow V, Beck D, Budnitz T, Miller DC, Potter J, Wears RL, et al. Transitions of Care Consensus policy statement: American College of Physicians, Society of General Internal Medicine, Society of Hospital Medicine, American Geriatrics Society, American College Of Emergency Physicians, and Society for Academic Emergency Medicine. J Hosp Med 2009;4:364-70.

13. Odell M. The patient's thoughts and feelings about their transfer from intensive care to the general ward. J Adv Nurs 2000;31:322-9.

14. Linton S, Grant C, Pellegrini J. Supporting families through discharge from PICU to the ward: the development and evaluation of a discharge information brochure for families. Intensive Crit Care Nurs 2008;24:329-37.

15. Parsons Leigh J, Brundin-Mather R, Zjadewicz K, Soo A, Stelfox HT. Improving transitions in care from intensive care units: development and pilot testing of an electronic communication tool for healthcare providers. J Crit Care 2020;56:265-72.

16. Watts RJ, Pierson J, Gardner H. How do critical care nurses define the discharge planning process? Intensive Crit Care Nurs 2005;21:39-46.

17. Davies H, McKenzie N, Williams TA, Leslie GD, McConigley $\mathrm{R}$, Dobb GJ, et al. Challenges during long-term follow-up of ICU patients with and without chronic disease. Aust Crit Care 2016;29:27-34.

18. de Grood C, Leigh JP, Bagshaw SM, Dodek PM, Fowler RA, Forster AJ, et al. Patient, family and provider experiences with transfers from intensive care unit to hospital ward: a multicentre qualitative study. CMAJ 2018;190:E669-76.

19. Graneheim UH, Lundman B. Qualitative content analysis in nursing research: concepts, procedures and measures to achieve trustworthiness. Nurse Educ Today 2004;24:105-12.

20. Lincoln YS, Lynham SA, Guba EG. Paradigmatic controversies, contradictions, and emerging confluences, revisited. In: Denzin NK, Lincoln YS, editors. The Sage handbook of qualitative research. Thousand Oaks: Sage; 2011. p. 97-128.

21. Lincoln YS, Guba EG. But is it rigorous? Trustworthiness and authenticity in naturalistic evaluation. New Dir Program Eval 1986;1986:73-84.

22. Engel HJ, Needham DM, Morris PE, Gropper MA. ICU early mobilization: from recommendation to implementation at three medical centers. Crit Care Med 2013;41(9 Suppl 1):S6980.

23. Pandullo SM, Spilman SK, Smith JA, Kingery LK, Pille SM, Rondinelli RD, et al. Time for critically ill patients to regain mobility after early mobilization in the intensive care unit and transition to a general inpatient floor. J Crit Care 2015; 30:1238-42.

24. Cullinane JP, Plowright CI. Patients' and relatives' experiences of transfer from intensive care unit to wards. Nurs Crit Care 2013;18:289-96.

25. Cypress BS. Transfer out of intensive care: an evidence-based literature review. Dimens Crit Care Nurs 2013;32:244-61.

26. Op 't Hoog SA, Dautzenberg M, Eskes AM, Vermeulen H, Vloet LC. The experiences and needs of relatives of intensive care unit patients during the transition from the intensive care unit to a general ward: a qualitative study. Aust Crit Care 2020;33: 526-32.

27. Forsberg A, Lindgren E, Engström Å. Being transferred from an intensive care unit to a ward: searching for the known in the unknown. Int J Nurs Pract 2011;17:110-6.

28. Chaboyer W, Kendall E, Kendall M, Foster M. Transfer out of intensive care: a qualitative exploration of patient and family perceptions. Aust Crit Care 2005;18:138-41, 143-5.

29. Field K, Prinjha S, Rowan K. 'One patient amongst many': a qualitative analysis of intensive care unit patients' experiences of transferring to the general ward. Crit Care 2008;12:R21.

30. Beard H. Does intermediate care minimize relocation stress for patients leaving the ICU? Nurs Crit Care 2005;10:272-8.

31. Keller G, Merchant A, Common C, Laizner AM. Patient experiences of in-hospital preparations for follow-up care at home. J Clin Nurs 2017;26:1485-94.

32. Deacon KS. Re-building life after ICU: a qualitative study of the patients' perspective. Intensive Crit Care Nurs 2012;28: 114-22.

33. Mitchell ML, Courtney M, Coyer F. Understanding uncertainty and minimizing families' anxiety at the time of transfer from intensive care. Nurs Health Sci 2003;5:207-17. 
34. Goossens E, Hilderson D, Moons P. Coaching through transition: a challenge for critical care nurses. Aust Crit Care 2012; 25:1-2.

35. Ågård AS, Egerod I, Tønnesen E, Lomborg K. From spouse to caregiver and back: a grounded theory study of post-intensive care unit spousal caregiving. J Adv Nurs 2015;71:1892-903.

36. Bouneb R, Mellouli M, Dardouri M, Soltane HB, Chouchene I, Boussarsar M. Determinants and outcomes associated with decisions to deny intensive care unit admission in Tunisian ICU. Pan Afr Med J 2018;29:176.

37. Halvorson S, Wheeler B, Willis M, Watters J, Eastman J, O'Donnell R, et al. A multidisciplinary initiative to standardize intensive care to acute care transitions. Int J Qual Health Care 2016;28:615-25.

38. Guest M. Patient transfer from the intensive care unit to a general ward. Nurs Stand 2017;32:45-51. 